\title{
ANÁLISE E COMPARAÇÃO DO PERFIL ANTROPOMÉTRICO E POSTURAL DE ADOLESCENTES COM SÍNDROME DE DOWN
}

Maria Tereza Artero Prado ${ }^{1,2}$, Aline da Silva Oliveira ${ }^{1}$, Caroline Ghisi Barboza Gomes ${ }^{1}$, Francis Lopes Pacagnelli ${ }^{1}$, Renata Calciolari Rossi e Silva ${ }^{1}$, Patrícia Mayumi Takamoto ${ }^{1}$, Carlos Bandeira de Mello Monteiro $^{2,3}$, Deborah Cristina Gonçalves Luiz Fernani ${ }^{1,2}$

${ }^{1}$ Universidade do Oeste Paulista - UNOESTE. Faculdade de Ciências da Saúde, Presidente Prudente, SP. ${ }^{2}$ Faculdade de Medicina do ABC. Laboratório de Escrita Científica, Santo André, SP. ${ }^{3}$ Universidade de São Paulo - USP. Escola de Artes, Ciências e Humanidades, São Paulo, SP. e-mail: mariatereza@unoeste.br.

\section{RESUMO}

O objetivo deste estudo foi analisar e comparar o perfil antropométrico e postural de adolescentes com Síndrome de Down (SD). A amostra foi composta por 36 indivíduos divididos em: Grupo Experimental (GE) com 18 sujeitos com SD (média de idade de 15,16 $\pm 3,51$ anos) e um Grupo Controle (GC) com 18 indivíduos com desenvolvimento típico (média de idade de 13,05 $\pm 2,26$ anos). Para classificação dos dados antropométricos foi utilizado o índice de massa corpórea pela idade (IMC/idade) e para análise da postura o Instrumento de Avaliação Postural. Ao comparar a classificação do IMC/idade entre os grupos, constatou-se que $66,67 \%$ do GC foram considerados eutróficos e $33,33 \%$ no GE como obesos. Ambos os grupos apresentaram alterações posturais, sendo significativa a presença de joelhos valgos e tronco em rotação à esquerda na vista anterior no GE. Enfatiza-se a importância da avaliação postural na SD para que possíveis alterações posturais sejam evitadas e minimizadas.

Palavras-chave: Síndrome de Down; Postura; Adolescente; Obesidade; Sobrepeso.

\section{ANALYSIS AND COMPARISON OF ANTHROPOMETRIC AND POSTURAL PROFILE IN ADOLESCENTS WITH DOWN SYNDROME}

\begin{abstract}
The aim of this study was to analyze and compare the postural and anthropometric profile of adolescents with Down Syndrome (DS). The sample consisted of 36 individuals divided into: Experimental Group (EG) with 18 individuals with DS (mean age 15.16 \pm 3.51 years) and a Control Group (CG) with 18 typically developing individuals (mean age $13.05 \pm 2.26$ years). For classification of the anthropometric data was used body mass index for age (BMI/age) and for analysis posture the Instrumento de Avaliação Postural. Comparing to the classification of BMI/age between the groups, it was found that $66.67 \%$ of the CG were considered eutrophic and $33.33 \%$ in EG as obese. Both groups showed postural changes, with a significant presence of valgus knees and trunk rotation to the left in the anterior view in EG. Emphasizes the importance of the postural evaluation in DS for possible postural changes are avoided and minimized.
\end{abstract}

Keywords: Down Syndrome; Posture; Adolescent; Obesity; Overweight. 


\section{INTRODUÇÃO}

A Síndrome de Down (SD) apresentou o primeiro registro no ano de $1866^{1}$ e pode ser caracterizada, em sua maioria, pela trissomia do cromossomo no par $21^{2}$, o qual ocasiona alterações no desenvolvimento motor, físico e intelectual ${ }^{3,4}$. Segundo Micheletto (2009) ${ }^{4}$, estima-se que no ano de 2005 nasceram 5.058 crianças com a SD e que aproximadamente existem cerca de 300 mil brasileiros com esta síndrome. Uma explicação para estes números expressivos seria o Brasil ser um país com território extenso, no qual ocorre às miscigenações entre as etnias, o que pode contribuir para a propagação do gene alterado ${ }^{5}$.

A obesidade apresenta-se prevalente nos sujeitos com SD sendo mais encontrada em adolescentes, quando comparadas ao restante da população ${ }^{6,7}$. Na SD, o mecanismo da obesidade não é totalmente explicado, porém, estudos sugerem alguns fatores tais como o hipotireoidismo ${ }^{8}$. Além disso, o ganho de peso corporal nesses indivíduos é superior ao ganho de estatura, o que resulta em um índice de massa corpórea elevado $^{9}$. Segundo a Organização Mundial da Saúde (OMS), a obesidade é um desafio de saúde pública, sendo uma doença que triplicou nas duas últimas décadas e continua a aumentar, associa-se a um aumento da morbidade e mortalidade, fator este influenciador direto na qualidade de vida ${ }^{10}$.
Um fator que tem sido investigado nos indivíduos com excesso de peso são as alterações posturais, na SD a maioria das vezes estas alterações posturais também provêm de frouxidão ligamentar e músculos hipotônicos. Quando associadas ao sobrepeso e obesidade seus sinais podem ser mais evidentes, caso não apresente diagnóstico nos estágios iniciais, estas alterações tornam-se irreversíveis e graves na idade adulta $^{11}$, as quais afetam negativamente a eficiência da musculatura envolvida e podem desencadear dor e outras condições musculoesqueléticas patológicas e nada estéticas ${ }^{12}$. Assim, faz-se necessária o uso de avaliação postural, para que se possa mensurar os desequilíbrios e planejar um tratamento específico para cada indivíduo, pois o objetivo desta análise é visualizar e determinar segmentos corporais que possam estar desalinhados ${ }^{13}$, para evitar ou diminuir a probabilidade e o progresso dessas alterações antes que se tornem irreversíveis e ocasionem outros distúrbios. Portanto, este estudo apresenta como objetivo, analisar e comparar o perfil antropométrico e postural de adolescentes com SD por avaliação visual e analítica.

\section{MÉTODOS}

Foram avaliados 36 indivíduos divididos em dois grupos: o Grupo Experimental (GE) composto por 18 sujeitos 
com SD (média de idade de 15,16 $\pm 3,51$ anos, sendo 6 indivíduos do sexo feminino e 12 do masculino) e que frequentavam uma Instituição de apoio à educação e saúde, situada na cidade de Presidente Prudente/SP e um Grupo Controle (GC) com 18 indivíduos com desenvolvimento típico (média de idade de $13,05 \pm 2,26$ anos, dos quais 7 eram do sexo feminino e 11 do masculino) selecionados aleatoriamente em uma instituição de ensino regular. Os critérios de inclusão para ambos os grupos foi 0 consentimento dos adolescentes para a participação no estudo e a autorização dos responsáveis. Foram excluídos os indivíduos que apresentavam outros diagnósticos neurológicos associados. Este estudo foi aprovado pelo Comitê de Ética em Pesquisa da Universidade do Oeste Paulista sob o número de protocolo 1155/12.

Inicialmente foram coletados os dados de idade e sexo, seguidos da verificação do peso, por uma balança digital da marca Plenna e da altura por um estadiômetro da marca Balmak ${ }^{\oplus}$ fixado na parede. Realizou-se a classificação do índice de massa corpórea pela idade (IMC/idade) de acordo com os gráficos do Sistema de Vigilância Alimentar e Nutricional (SISVAN) $(2007)^{14}$, os quais apresentam como objetivo a detecção de situações de risco nutricional pelos seguintes critérios: Escore-z $<$ que -3 significam Magreza acentuada; Escore-z > que -3 e $<$ que -2 = Magreza; Escore- $z>$ que 2 e $<$ que +1 = Eutrofia; Escore- $z>$ que +1 e $<$ que +2 = Sobrepeso; Escore-z $>$ que +2 e $<$ que $+3=$ Obesidade; Escore- $z>$ que $+3=$ Obesidade grave.

Para análise do perfil postural os indivíduos foram posicionados em um quadrado demarcado no chão, onde eram tiradas fotografias nas vistas anteriores, perfis e posteriores ${ }^{15}$, por uma câmera digital da marca Sony ${ }^{\oplus}$ DSC-W320 com 14.1 mega pixels, fixada sobre tripé da marca $W^{\oplus}$, modelo t3330 com altura de um metro e trinta centímetros do solo e distância do indivíduo a ser avaliado de dois metros ${ }^{16}$. Em seguida utilizou-se do Instrumento de Avaliação Postural (IAP) de Liposcki et. al $(2007)^{17}$, sendo um formulário de análise postural preenchido conforme a análise visual direta das avaliadoras, também posicionadas a dois metros do indivíduo. Posteriormente, foi realizada uma análise de caráter comprobatório para comparação e confirmação da avaliação visual presencial anteriormente registrada em associação a análise das fotografias. Para verificar as principais alterações posturais presente em cada grupo (GE e GC), utilizou-se da frequência de no mínimo $50 \%$ do grupo com determinada alteração.

Após a conclusão das avaliações, os resultados foram entregues aos responsáveis pelos participantes com o intuito de que com 
o conhecimento desses achados, possam buscar um tratamento adequado e acompanhar a sua progressão ${ }^{15}$. Também foram sugeridos locais acessíveis que pudessem realizar o tratamento conforme possibilidade dos mesmos. Os dados encontrados foram expressos em valores absolutos e percentuais. Os índices de massa corporal/idade aferidos para os grupos estudados foram comparados pelo teste $t$ não pareado e para determinar se as frequências dos parâmetros aferidos diferiram entre o GC e o GE recorreu-se ao teste exato de Fisher. Todas as análises foram conduzidas no Software Biostat 5.0 e o valor de significância adotado foi de 5\%.

\section{RESULTADOS}

Mediante a análise dos dados antropométricos dos grupos, $\mathrm{O} \quad \mathrm{GE}$ apresentou a média do IMC/idade de $26,90 \pm 5,75 \mathrm{Kg} / \mathrm{m}^{2}$ e o GC de $19,62 \pm 4,46$ $\mathrm{Kg} / \mathrm{m}^{2}$, com diferença significativa $(p=0,0006)$. A maior frequência da classificação do IMC/idade no GC foi de eutrofia com 66,67\%, já no GE foi de obesidade em $33,33 \%$ dos indivíduos. $\mathrm{Na}$ Tabela 1 estão apresentados todos os valores referentes à classificação.

Tabela 1. Valores absolutos e relativos da classificação do IMC/Idade

\begin{tabular}{ccccc}
\hline \multirow{2}{*}{ Categoria } & \multicolumn{2}{c}{$\mathbf{G C}(\mathbf{n}=\mathbf{1 8})$} & \multicolumn{2}{c}{$\mathbf{G E}(\mathbf{n}=\mathbf{1 8})$} \\
\cline { 2 - 5 } & $\mathrm{n}$ & $\%$ & 0 & $\%$ \\
\hline Magreza acentuada & 2 & $11,11 \%$ & 0 & $0,00 \%$ \\
Magreza & 2 & $11,11 \%$ & 5 & $0,00 \%$ \\
Eutrofia & 12 & $66,67 \%$ & 4 & $27,78 \%$ \\
Sobrepeso & 1 & $5,56 \%$ & 6 & $22,22 \%$ \\
Obesidade & 1 & $5,56 \%$ & 3 & $33,33 \%$ \\
Obesidade grave & 0 & $0,00 \%$ & & $16,67 \%$ \\
\hline
\end{tabular}

$\mathrm{GC}=$ Grupo Controle; GE=Grupo Experimental; \%=porcentagem.

$\mathrm{Na}$ análise do perfil postural, foram encontradas alterações posturais em todas as vistas e em ambos os grupos, porém, apenas na vista anterior obteve-se significância entre os mesmos, sendo as alterações: tronco em rotação esquerda e joelhos valgos. Em relação às alterações posturais mais encontradas, foi observado em $50 \%$ do GE: ombros protusos, ombro elevado à esquerda, joelhos valgos, cintura pélvica em anteversão, pés planos e valgos. Porém o GC não apresentou principais alterações posturais como o $\mathrm{GE}$, fato visto pela dispersão das alterações analisadas, sendo observadas nas Tabelas 2, 3 e 4. Também detectou-se a presença de abdome protuso em ambos os grupos (GE=10; GC=12). 
Tabela 2. Alterações posturais visualizadas na vista anterior em ambos os grupos

\begin{tabular}{|c|c|c|c|}
\hline Parâmetros & $G C(n=18)$ & GE $(n=18)$ & $p$ \\
\hline Cabeça inclinada à direita & $3 / 18(16,67 \%)$ & $5 / 18(27,78 \%)$ & 1,0000 \\
\hline Cabeça inclinada à esquerda & $2 / 18(11,11 \%)$ & $4 / 18(22,22 \%)$ & 1,0000 \\
\hline Rotação de cabeça à esquerda & $1 / 18(5,56 \%)$ & $0 / 18(0,00 \%)$ & 1,0000 \\
\hline Rotação de cabeça à direita & $1 / 18(5,56 \%)$ & $1 / 18(5,56 \%)$ & 1,0000 \\
\hline Ombro elevado à direita & $7 / 18(38,89 \%)$ & $7 / 18(38,89 \%)$ & 0,6370 \\
\hline Ombro elevado à esquerda & $7 / 18(38,89 \%)$ & $9 / 18(50,00 \%)$ & 1,0000 \\
\hline Triângulo de Tales assimétrico à direita & $3 / 18(16,67 \%)$ & $4 / 18(22,22 \%)$ & 1,0000 \\
\hline Triângulo de Tales assimétrico à esquerda & $5 / 18(27,78 \%)$ & $6 / 18(33,33 \%)$ & 0,6148 \\
\hline Tronco em rotação à direita & $1 / 18(5,56 \%)$ & $3 / 18(16,67 \%)$ & 0,1666 \\
\hline Tronco em rotação à esquerda & $4 / 18(22,22 \%)$ & $7 / 18(38,89 \%)$ & $0,0114^{*}$ \\
\hline Crista ilíaca assimétrica à direita & $1 / 18(5,56 \%)$ & $4 / 18(22,22 \%)$ & 1,0000 \\
\hline Crista ilíaca assimétrica à esquerda & $5 / 18(27,78 \%)$ & $4 / 18(22,22 \%)$ & 0,2777 \\
\hline Quadril em rotação interna à direita & $0 / 18(0,00 \%)$ & $0 / 18(0,00 \%)$ & 1,0000 \\
\hline Quadril em rotação interna à esquerda & $3 / 18(16,67 \%)$ & $0 / 18(0,00 \%)$ & 1,0000 \\
\hline Quadril em rotação externa à direita & $2 / 18(11,11 \%)$ & $3 / 18(16,67 \%)$ & 0,3137 \\
\hline Quadril em rotação externa à esquerda & $1 / 18(5,56 \%)$ & $3 / 18(16,67 \%)$ & 1,0000 \\
\hline Quadril em rotação interna dos dois membros & $1 / 18(5,56 \%)$ & $1 / 18(5,56 \%)$ & 1,0000 \\
\hline Quadril em rotação externa dos dois membros** & - & $7 / 18(38,89 \%)$ & - \\
\hline Joelho valgo direito & $2 / 18(0,00 \%)$ & $0 / 18(0,00 \%)$ & 1,0000 \\
\hline Joelho valgo esquerdo & $0 / 18(0,00 \%)$ & $0 / 18(0,00 \%)$ & 1,0000 \\
\hline Joelho varo direito & $0 / 18(11,11 \%)$ & $0 / 18(0,00 \%)$ & 1,0000 \\
\hline Joelho varo esquerdo & $2 / 18(27,78 \%)$ & $0 / 18(0,00 \%)$ & 1,0000 \\
\hline Joelhos valgos & $5 / 18(27,78 \%)$ & $9 / 18(50,00 \%)$ & $0,0294^{*}$ \\
\hline Joelhos varos & $5 / 18(27,78 \%)$ & $1 / 18(5,56 \%)$ & 0,2777 \\
\hline
\end{tabular}

$\mathrm{GC}=\mathrm{Grupo}$ Controle; GE=Grupo Experimental; \%=porcentagem; * $\mathrm{p}<0,05$; ** mensurado apenas para o GE.

Contudo, como nas vistas de perfis (direita e esquerda) a avaliação postural foi idêntica para ambos os perfis em todos os indivíduos, adotou-se a referência de apenas um perfil para demonstração. Na análise das vistas perfil e posterior, não foram encontradas alterações com significância entre os grupos estudados (Tabelas 3 e 4). 
Tabela 3. Alterações posturais visualizadas na vista de perfil em ambos os grupos

\begin{tabular}{lccc}
\hline \multicolumn{1}{c}{ Parâmetros } & $\mathbf{G C}(\mathbf{n = 1 8})$ & $\mathbf{G E}(\mathbf{n = 1 8})$ & $\boldsymbol{p}$ \\
\hline Cabeça projetada para frente & $1 / 18(5,56 \%)$ & $3 / 18(16,67 \%)$ & 0,1666 \\
Cabeça projetada para trás & $0 / 18(0,00 \%)$ & $0 / 18(0,00 \%)$ & 1,0000 \\
Ombros protusos & $6 / 18(33,33 \%)$ & $12 / 18(66,67 \%)$ & 0,3440 \\
Ombros retraídos & $2 / 18(11,11 \%)$ & $0 / 18(0,00 \%)$ & 1,0000 \\
Coluna cervical em hiperlordose & $1 / 18(5,56 \%)$ & $2 / 18(11,11 \%)$ & 0,1111 \\
Coluna cervical em retificação & $0 / 18(0,00 \%)$ & $0 / 18(0,00 \%)$ & 1,0000 \\
Coluna torácica em hipercifose & $3 / 18(16,67 \%)$ & $0 / 18(0,00 \%)$ & 1,0000 \\
Coluna torácica retificada & $0 / 18(0,00 \%)$ & $0 / 18(0,00 \%)$ & 1,0000 \\
Coluna lombar em hiperlordose & $6 / 18(33,33 \%)$ & $7 / 18(38,89 \%)$ & 0,6266 \\
Coluna lombar em retificação & $1 / 18(5,56 \%)$ & $0 / 18(0,00 \%)$ & 1,0000 \\
Cintura pélvica em anteversão & $8 / 18(44,44 \%)$ & $10 / 18(55,56 \%)$ & 1,0000 \\
Cintura pélvica em retroversão & $1 / 18(5,56 \%)$ & $0 / 18(0,00 \%)$ & 1,0000 \\
Joelhos hiperextendidos & $1 / 18(5,56 \%)$ & $2 / 18(11,11 \%)$ & 1,0000 \\
Joelhos hiperfletidos & $0 / 18(0,00 \%)$ & $2 / 18(11,11 \%)$ & 1,0000 \\
\hline
\end{tabular}

$\mathrm{GC}=$ Grupo Controle; GE=Grupo Experimental; \%=porcentagem.

Tabela 4. Alterações posturais visualizadas na vista posterior em ambos os grupos

\begin{tabular}{lccc}
\hline \multicolumn{1}{c}{ Parâmetros } & GC (n=18) & GE (n=18) & $p$ \\
\hline Escápula alada direita & $0(0,00 \%)$ & $1(5,56 \%)$ & 1,0000 \\
Escápula alada esquerda & $0(0,00 \%)$ & $0(0,00 \%)$ & 1,0000 \\
Escápula retraída direita & $0(0,00 \%)$ & $0(0,00 \%)$ & 1,0000 \\
Escápula retraída esquerda & $0(0,00 \%)$ & $0(0,00 \%)$ & 1,0000 \\
Escápula alada ambos os lados & $8(44,44 \%)$ & $4(22,22 \%)$ & 0,2745 \\
Coluna vertebral escoliose em "S" & $6(33,33 \%)$ & $3(16,67 \%)$ & 0,5147 \\
Coluna vertebral escoliose em "S" invertido & $0(0,00 \%)$ & $0(0,00 \%)$ & 1,0000 \\
Coluna vertebral escoliose em "C" & $5(27,78 \%)$ & $4(22,22 \%)$ & 0,2777 \\
Pregas glúteas assimétricas à direita & $8(44,44 \%)$ & $6(33,33 \%)$ & 1,0000 \\
Pregas glúteas assimétricas à esquerda & $5(27,78 \%)$ & $3(16,67 \%)$ & 0,5220 \\
Pé esquerdo plano & $8(44,44 \%)$ & $18(100,00 \%)$ & 1,0000 \\
Pé esquerdo cavo & $2(11,11 \%)$ & $0(0,00 \%)$ & 1,0000 \\
Pé esquerdo varo & $1(5,56 \%)$ & $1(5,56 \%)$ & 1,0000 \\
Pé esquerdo valgo & $2(11,11 \%)$ & $14(77,78 \%)$ & 1,0000 \\
Pé direito plano & $8(44,44 \%)$ & $18(100,00 \%)$ & 1,0000 \\
Pé direito cavo & $2(11,11 \%)$ & $0(0,00 \%)$ & 1,0000 \\
Pé direito valgo & $0(0,00 \%)$ & $15(83,33 \%)$ & 1,0000 \\
Pé direito varo & $3(16,67 \%)$ & $1(5,56 \%)$ & 1,0000 \\
\hline
\end{tabular}

GC=Grupo Controle; GE=Grupo Experimental, \%=porcentagem.

\section{DISCUSSÃO}

No presente estudo foi possível verificar que houve diferença significativa entre os grupos em relação ao IMC/Idade e as alterações posturais observadas na vista anterior. Com base nos dados encontrados na literatura, observa-se que os indivíduos com SD apresentam maior porcentagem de excesso de peso quando comparadas com a população sem a síndrome ${ }^{18}$. Em um estudo realizado por Cronk $^{9}$ foi observado que indivíduos com SD tendem a ter um aumento 
do índice de massa corpórea, com prevalência de sobrepeso e obesidade, fato que corrobora com este estudo, no qual a prevalência dos indivíduos analisados do GE foi de $33,33 \%$ de obesos. Já em relação ao GC, os indivíduos classificados como obesos foi de apenas 5,56\%, o que difere do estudo de Neutzling et al. $(2000)^{18}$, que avaliaram adolescentes com idade entre 10 e 19 anos e obtiveram $7,7 \%$ de sobrepeso e obesidade entre eles.

$$
\text { Guadagnin et al. (2012) }
$$
encontraram em seu estudo que os desvios posturais fazem parte da vida de grande parte da população adulta mundial e a presença deles também está sendo investigada em crianças e adolescentes. Neste estudo foi detectado em todos os indivíduos algum tipo de alteração postural e as duas alterações que atingiram mais de $50 \%$ de um dos grupos da amostra (GE) foi a protusão e a elevação do ombro (Tabelas 3 e 4). Este achado concorda parcialmente com a pesquisa de Molinari et al. $(2010)^{11}$ que avaliou crianças com SD obesas e eutróficas, não verificou a presença de ombros protusos e constatou a presença de $80 \%$ de sua amostra com ombro elevado, que pode ser decorrente do desequilíbrio muscular generalizado característico em indivíduos com SD. Também foi encontrado neste estudo a presença de joelhos valgos na maioria dos indivíduos com SD classificados como obesos. Pinto et al. $(2006)^{20}$ relataram que pré adolescentes obesos apresentam maior taxa desta alteração.

Os dados encontrados nesta pesquisa com relação à presença de pé plano em todos os indivíduos do GE e em oito do GC, apresentou uma concordância com o estudo de Molinari et al.(2010) ${ }^{11}$, no qual as crianças com SD avaliadas e classificadas como obesas apresentaram maior índice de pé plano do que as eutróficas e ao comparar esses achados, sugeriu que o tipo físico influencia no tipo de pé desses sujeitos. Também foi visto a presença de pés valgos na maioria dos indivíduos do $\mathrm{GE}$, dado este que corrobora com a pesquisa de Matos $(2005)^{21}$, o qual relatou que sujeitos com SD apresentam muitas alterações ortopédicas, dentre elas os pés planos valgos, que pode estar relacionado à hiperfrouxidão ligamentar generalizada que estes indivíduos apresentam.

Além das alterações acima citadas, identificou-se o abdome protuso na maioria dos indivíduos avaliados tanto do GC (12) como no GE (10). Em um estudo foi verificado que a hiperlordose lombar foi à alteração mais frequente observada, uma vez que está associada à anteroversão pélvica e abdome protuso $^{11}$, dado que também foi expressivo nesta pesquisa. Além deste fato, no atual estudo foram encontradas essas alterações pélvicas também nos indivíduos 
eutróficos do GC, que pode ser explicado pela diminuição de força muscular, associado à inatividade física. Segundo Arruda e Simões $(2006)^{22}$, alunos regulares apresentam uma prevalência de hiperlordose lombar quando classificados com sobrepeso e obesidade.

Sabe-se que a postura inadequada tende a instalar-se precocemente, sendo moldadas desde a infância até adolescência. Este é um dos motivos para enfatizar a importância dessas avaliações, que detectem alterações posturais e que sejam abordadas preventivamente ${ }^{23}$.

Encontrou-se como limitação do estudo o número reduzido de indivíduos com SD, situação que sugere a realização de outras pesquisas com amostra maior, além da análise interna dos elementos de cada grupo.

\section{CONCLUSÃO}

Portanto, ao analisar e comparar os perfis antropométricos e posturais dos adolescentes de ambos os grupos, foi constatado que os adolescentes com SD foram predominantemente classificados como obesos e os com desenvolvimento típico como eutróficos. Em ambos os grupos foram encontradas alterações posturais, porém, foi observada maior frequência de determinadas alterações no grupo experimental como: ombros protusos, ombro elevado à esquerda, joelhos valgos, cintura pélvica em anteversão, pés planos e valgo, com valor adotado igual ou maior que $50 \%$.

\section{REFERÊNCIAS}

1. Baum RA, Nash PL, Foster JEA, Spader $M$, Ratliff-Schaub K, Coury D. Primary Care of Children and Adolescents with Down Syndrome: An Update. Curr Probl Pediatr Adolesc Health Care 2008 Sep; 241-61. http://dx.doi.org/10.1016/i.cppeds.2008.07. $\underline{001}$

2. Mendes CC, Biselli JM, Zampieri BL, Bertollo EMG, Eberlin MN, Haddad R, et al.19-base pair deletion polymorphism of the dihydrofolate reductase (DHFR) gene: maternal risk of Down syndrome and folate metabolism. São Paulo Med J 2010;128(4):215-8.

http://dx.doi.org/10.1590/S1516$\underline{31802010000400008}$

3. Gorla JL, Duarte E, Costa LT, Freire F. Crescimento de crianças e adolescentes com Síndrome de Down - Uma breve revisão de literatura. Rev Bras Cineantropom Desempenho Hum 2011;13(3):230-237.

4. Micheletto MRD, Amaral VLAR, Valerio NI, Conte ACF. Adesão ao tratamento após aconselhamento genético na Síndrome de Down. Psicol Estud 2009;14(3):491-500. http://dx.doi.org/10.1590/S1413-

$\underline{73722009000300010}$

5. Bertelli ECP, Biselli JM, Bonfim D, Bertollo EMG. Clinical profile of children with Down syndrome treated in a genetics outpatient service in the Southeast of Brazil. Rev Assoc Med Bras 2009;55(5):547-52. http://dx.doi.org/10.1590/S010442302009000500017

6. Grammatikopoulou MG, Manai A, Tsigga M, Tsiligiroglou-Fachantidou A, GalliTsinopoulou A, Zakas A. Nutrient intake and anthropometry in children and adolescents 
with Down syndrome - a preliminary study. Dev Neurorehabil 2008 Oct;11(4):260-67. http://dx.doi.org/10.1080/175184208025255 $\underline{26}$

7. Melville CA, Cooper SA, McGrother CW, Thorp CF, Collacott R. Obesity in adults with Down syndrome: a case-control study. J Intellect Disabil Res 2004; 49(2):125-33. http://dx.doi.org/10.1111/j.1365$\underline{2788.2004 .00616 . x}$

8. Coleman M. Thyroid dysfunction in Down's syndrome: a review. Downs Syndr Res Pract 1994 Oct;2(3):112-15. http://dx.doi.org/10.3104/reviews.40

9. Cronk C, Crocker AC, Pueschel SM, Shea AM, Zackai E, Pickens $G$ et al. Growth charts for children with Down syndrome: 1 month to 18 years of age. Pediatr 1988; 81(1):102-110.

10. Branca $F$, Nikogosian $H$, Lobstein $T$. The challenge of obesity in the WHO European Region and the strategies for response. World Health Organization 2007.

11. Molinari VS, Massuia OAF. Análise da postura e apoio plantar de crianças portadoras de Síndrome de Down consideradas obesas. J Health Sci Inst 2010;28(4):345-7345.

12. James $H$, Castaneda L, Miller ME, Findley T. Rolfing structural integration treatment of cervical spine dysfunction. J Body Mov Ther 2009;13(3):229-38.

http://dx.doi.org/10.1016/i.jbmt.2008.07.00 $\underline{2}$

13. Mansoldo CA, Nobre ADP. Avaliação postural em nadadores federados praticantes do nado borboleta nas provas de 100 e 200 metros. O mundo da saúde. 2007 out/dez; 31(4):511-520.

14. SISVAN (Sistema de Vigilância Alimentar e Nutricional). Curvas de Crescimento da
Organização Mundial da Saúde-OMS. [on line]: Brasil 2007. Disponível em: $<$ http://nutricao.saude.gov.br/sisvan.

Php?Conteudo=curvas_cresc_oms $>$ [2013 abr 02]

15. Sanchez HM, Barreto RR, Baraúna MA, Canto RST, Morais EG. Avaliação postural de indivíduos portadores de deficiência visual através da Biofotogrametria Computadorizada. Fisioter Mov 2008;21(2):11-20.

16. Souza JA, Pasinato F, Basso D, Corrêa ECR, Silva AMT. Biofotogrametria confiabilidade das medidas do protocolo do software para avaliação postural (SAPO). Rev Bras Cineantropom Desempenho Hum 2011;13(4):299-305.

17. Liposcki DB, Rosa Neto F, Savall AC. Validação do conteúdo do Instrumento de Avaliação Postural - IAP. Rev Digital Efdeportes Buenos Aires 2007;12(109). Disponível em: $<$ http://www.efdportes.com/edf109/validaca o-do-conteudo-do-instrumento-de-avaliacaopostural.htm> [2013 mar 16]

18. Neutzling MB, Taddei JAAC, Rodrigues $E M$, Sigulem DM. Overweight and obesity in Brazilian adolescents. Int J Obes Relat Metab Disord 2000;24(7):869-74. http://dx.doi.org/10.1038/sj.ijo.0801245

19. Guadagnin EC, Matheus SC. Prevalência de desvios posturais de coluna vertebral em escolares. Rev Bras Ciênc Saúde 2012; 10(31): 31-7.

http://dx.doi.org/10.13037/rbcs.vol10n31.14 $\underline{63}$

20. Pinto ALS, Holanda PMB, Radu AS, Villares SMF, Lima FR. Musculoskeletal findings in obese children. J Paediatr Child Health 2006;42(6):341-4. http://dx.doi.org/10.1111/i.1440$\underline{1754.2006 .00869 . x}$ 
21. Matos MA. Instabilidade atlantoaxial e hiperfrouxidão ligamentar na Síndrome de Down. Acta Ortop Bras 2005;13(4):165-7. http://dx.doi.org/10.1590/S1413-

78522005000400001

22. Arruda MF, Simões MJS. Caracterização do excesso de peso na infância e sua influência sobre o sistema musculoesquelético de escolares de Araraquara-SP. Braz $\mathrm{J}$ of Food and Nutr 2006;7(4):413-418.

23. Santos, CIS et al. Ocorrência de desvios posturais em escolares do ensino público fundamental de Jaguariúna, São Paulo. Rev Paul Pediatr 2009;27(1)74-80. http://dx.doi.org/10.1590/s0103-

05822009000100012

Recebido para publicação em 30/07/2014

Revisado em 02/08/2014

Aceito em 03/08/2014 